\title{
639 PIK3IP1/TRIP IMMUNE REGULATION ON CD8+ T CELLS RESTRICTS ANTI-TUMOR IMMUNITY
}

Benjamin Murter*, Hridesh Banerjee, Andrea Szymczak-Workman, Lawrence Kane. University of Pittsburgh, Pittsburgh, PA, United States

Background The signaling pathways involving phosphoinositide-3-kinases (PI3Ks) are highly conserved and tightly regulated to influence the activation, proliferation, and survival of all cell types. PI3K signaling plays a major role in $\mathrm{T}$ cell responses to antigen due to its position directly downstream

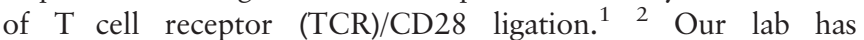
recently shown that the cell surface protein TrIP (Transmembrane Inhibitor of PI3K, gene name: Pik3ip1) has a distinctly high expression on $\mathrm{T}$ cells and is capable of downregulating PI3K signaling in CD4 $+\mathrm{T}$ cells, acting as a negative regulator of $\mathrm{T}$ cell immune responses. ${ }^{3}{ }^{4}$ These studies revealed that CD4+ $\mathrm{T}$ cells lacking TrIP expression exhibit a more Th1 inflammatory phenotype compared to WT $\mathrm{T}$ cells, both in vivo and in vitro. ${ }^{3}$ These data have led us to propose that TrIP restricts the inflammatory activity of $\mathrm{T}$ cells more generally, including $\mathrm{CD} 8+\mathrm{T}$ cells, and that targeting/knockout of this negative regulator may promote anti-tumor immunity.

Methods Using a conditional TrIP knockout mouse model developed in our lab, we have performed syngeneic tumor challenges in CD8 + T cell-specific TrIP knockout mice (TrIPfl/ fle8icre). We have also characterized the tumor immune infiltrate of these mice to understand the impact of $\mathrm{T}$ cell-specific TrIP deficiency on the immune landscape.

Results Our data thus far show that CD8 + T cell-specific TrIP knockout mice (TrIPfl/flE8icre) are resistant to growth of syngeneic tumors. In addition to increased tumor resistance, we have also found that tumors harvested from our TrIPfl/flE8icre knockout mice contain twice as many infiltrating $\mathrm{T}$ cells compared to their WT counterparts. We also found that CD8 $+\mathrm{T}$ cells appeared to be the main drivers of this increased $\mathrm{T}$ cell infiltration, as their frequency was double that of the CD4+ population in tumors transplanted into TrIP KO mice.

Conclusions We describe data demonstrating that TrIP, a relatively novel PI3K inhibitor, plays a significant role in the antitumor immune activity of CD8 $+\mathrm{T}$ cells. Our that CD8 $+\mathrm{T}$ cell-specific TrIP knockout mice are resistant to tumor challenge and show more robust tumor $\mathrm{CD} 8+\mathrm{T}$ cell infiltrate. With these data, we are excited to propose TrIP as a potential future immunotherapeutic target worthy of continued investigation.

\section{REFERENCES}

1. Okkenhaug $\mathrm{K}$, Turner M, Gold MR. PI3K signaling in B cell and T cell biology. Front Immunol 2014:5:557. doi:10.3389/fimmu.2014.00557

2. Kane LP, Weiss A. The PI-3 kinase/Akt pathway and T cell activation: pleiotropic pathways downstream of PIP3. Immunol Rev 2003;192:7-20. doi:10.1034/ j.1600-065X.2003.00008.X

3. Uche UU, Piccirillo AR, Kataoka $S$, et al. PIK3IP1/TrIP restricts activation of $T$ cells through inhibition of PI3K/Akt. J Exp Med 2018;215:3165-3179. doi:10.1084/ jem.20172018

4. DeFrances MC, Debelius DR, Cheng J, Kane LP. Inhibition of T-cell activation by PIK3IP1. Eur J Immunol 2012;42:2754-2759. doi:10.1002/eji.201141653

http://dx.doi.org/10.1136/jitc-2021-SITC2021.639 